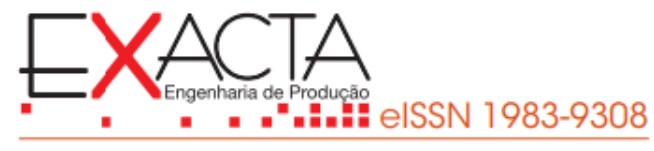

\title{
ESTUDO DA IMPLEMENTAÇÃO DO PRICING DINÂMICO EM UMA EMPRESA COM MODELO DE RECEITA RECORRENTE ATRAVÉS DA SEGMENTAÇÃO DE CLIENTES
}

\section{STUDY OF THE IMPLEMENTATION OF DYNAMIC PRICING IN A COMPANY WITH A RECURRING REVENUE MODEL THROUGH CUSTOMER SEGMENTATION}

Recebido em: 05 mar. 2020

Aprovado em: 03 jun. 2020

Versão do autor aceita publicada online: 03 jun. 2020

Publicado online: 18 jun. 2021

\section{Como citar esse artigo - American Psychological Association (APA):}

Rothbarth, C. S., Müller, M., Lerman, L. V., \& Sander, N. E. (2021, out./dez.). Estudo da implementação do pricing dinâmico em uma empresa com modelo de receita recorrente através da segmentação de clientes. Exacta. 19(4), 843-863. https://doi.org/10.5585/exactaep.2021.16754.

Submeta seu artigo para este periódico $\beta$

Dados Crossmark 


\title{
ESTUDO DA IMPLEMENTAÇÃO DO PRICING DINÂMICO EM UMA EMPRESA COM MODELO DE RECEITA RECORRENTE ATRAVÉS DA SEGMENTAÇÃO DE CLIENTES
}

\author{
STUDY OF THE IMPLEMENTATION OF DYNAMIC PRICING IN A COMPANY WITH A \\ RECURRING REVENUE MODEL THROUGH CUSTOMER SEGMENTATION
}

\author{
iD Catherine Sudbrack Rothbarth ${ }^{1}$ \\ iD Marcine Müller² \\ iD Laura Visintainer Lerman \\ iD Natália Eloísa Sander ${ }^{4}$
}

${ }^{1}$ Graduanda em Engenheira de Produção Universidade Federal do Rio Grande do Sul - UFRGS Porto Alegre, Rio Grande do Sul - Brasil. catherine.rothbarth@gmail.com

${ }^{2}$ Graduanda em Engenheira de Produção Universidade Federal do Rio Grande do Sul - UFRGS Porto Alegre, Rio Grande do Sul - Brasil. marcinemuller@hotmail.com

${ }^{3}$ Mestre em Engenharia de Produção Universidade Federal do Rio Grande do Sul - UFRGS Porto Alegre, Rio Grande do Sul - Brasil. lauravlerman@gmail.com

${ }^{4}$ Mestre em Engenharia de Produção Universidade Federal do Rio Grande do Sul - UFRGS Porto Alegre, Rio Grande do Sul - Brasil. nataliaesander@gmail.com

Recebido em: 05 mar. 2020

Aprovado em: 03 jun. 2020
Resumo: A formação do preço dos produtos e serviços constitui-se em uma decisão estratégica para as organizações. O processo de decisão de preço envolve uma série de variáveis complexas e interligadas, as quais devem ser entendidas como instrumento de gestão. Nesse contexto, este artigo busca revelar a necessidade de subordinar a precificação aos objetivos estratégicos e sugerir uma aplicação de pricing dinâmico para uma empresa de comunicação através da segmentação de clientes, uma vez que a empresa utiliza práticas superficiais e não justificáveis de gestão de preços. Sendo assim, além de perder qualidade no processo de tomada de decisão, seu modelo de aplicação de preços encontra-se defasado em relação ao dinamismo exigido pelo mercado. Trata-se de uma pesquisa que se baseia na revisão literária e na aplicação real da estratégia de preços mais adequada ao caso da empresa. Os resultados revelam as vantagens da gestão de preços embasada no planejamento estratégico.

Palavras-chave: Pricing dinâmico. Planejamento estratégico. Segmentação de clientes. Modelo de receita recorrente.

Abstract: The formation of the price of products and services is a strategic decision for organizations. The price decision process involves a series of complex and interconnected variables, which must be understood as a management tool. In this context, this article seeks to reveal the need to subordinate pricing to strategic objectives and to suggest a dynamic pricing application for a communication company through customer segmentation, since the company uses superficial and unjustified price management practices. Thus, in addition to losing quality in the decision-making process, its price application model is outdated in relation to the dynamism required by the market. This research is based on literary review and the real application of the most appropriate pricing strategy to the company's case. The results reveal the advantages of price management based on strategic planning.

Keywords: Dynamic pricing. Strategic planning. Customer segmentation. Recurring revenue model. 


\section{Introdução}

A concorrência pelo mercado consumidor no qual as organizações estão submetidas é dinâmica e, dentro dessa conjuntura, um resultado satisfatório está ligeiramente correlacionado à habilidade de gerenciar, analisar e prever os custos do negócio para criar ou sustentar vantagens competitivas. Isso ressalta, conforme destacam Ramos, Maia e Bornia (2005), a importância da tomada de decisão correta envolvendo o elemento preço - em relação à sua estrutura, gerenciamento, e utilização - para que os objetivos de uma empresa, a médio e a longo prazo, conforme definidos previamente no seu Planejamento Estratégico, sejam alcançados.

Montes e Chaves (2017) afirmam que a integração do mundo digital ao ambiente dos negócios contribuiu para a geração de um novo perfil de consumidor, mais conectado, maduro e criterioso no que se refere aos seus hábitos. Nesse contexto, surgem novas alternativas que buscam adequar o mercado à dinamicidade exigida pelo cliente. Uma delas é o chamado pricing dinâmico, em que a gestão dos preços possibilita a flexibilização da relação de valor entre produto e cliente. Torna-se possível, dessa forma, oferecer o mesmo item ou serviço por preços diferentes, de acordo com as necessidades características de cada perfil comprador.

Para que um perfil de cliente seja delimitado, é preciso que as empresas entendam as necessidades reais de seus consumidores, como forma de acompanhar o mercado e manter sua competitividade. Nesse contexto, surge a estratégia de segmentação de clientes, que significa a classificação dos clientes de uma empresa - os quais se almeja alcançar ou servir - em determinados grupos, para que se atue em diferentes objetivos (Abrate, Fraquelli, \& Viglia, 2012). Esses diferentes perfis de consumidores encontrados demonstram necessidades distintas, como a forma de relacionamento ou o preço do produto a ser oferecido. A partir do momento em que a empresa conhece os segmentos com os quais trabalha, é possível customizar seu produto ou serviço, e passar a expandir a sua carteira de clientes (Ayerbe, Cirion, Torres, Gil \& Laka, 2014; Raddats, Burton, \& Ashman, 2015).

Pricing dinâmico é um tema crescente na literatura, visto que as empresas inicialmente não conhecem os valores dos diferentes recursos (por exemplo, implantação de um novo sistema produtoserviço), mas podem aprender os valores dos recursos com base em vendas anteriores (Cohen, Lobel \& Paes, 2020). No entanto, normalmente, o princing dinâmico é abordado no sistema elétrico, em que a tarifa elétrica varia de acordo com demanda de energia, e os estudos na área focam em analisar a efetividade do princing dinâmico (Karlsen, Hamdy \& Attia, 2020). Portanto, mesmo que o pricing dinâmico seja fundamental para o desenvolvimento de fluxo de receitas sustentável, sua relação com o planejamento de negócios e com o modelo de negócios ainda foi pouco explorada na literatura, o que caracteriza uma lacuna de pesquisa. Dessa forma, objetivo deste trabalho é identificar e sugerir uma estrutura para gerenciar a precificação que traga vantagens tangíveis ao negócio. Busca-se, assim, 
definir a melhor prática a ser adotada, a partir de valores dos produtos previamente determinados. Para a escolha da estratégia de preço a ser seguida, deve-se realizar a revisão do Planejamento Estratégico e do modelo de receita da empresa, de modo a se certificar quais ações estão alinhadas aos objetivos da organização e à estrutura do negócio. Por fim, pretende-se experimentar a implementação da estratégia de pricing dinâmico que melhor se adeque ao negócio, e, em seguida mensurar e discutir os resultados.

Além desta introdução, a estrutura deste artigo contempla uma seção 2, a qual se destina à revisão da literatura. Esse item traz um compilado sobre a teoria necessária para o desenvolvimento da metodologia e resultados, ou seja, desdobra temas sobre o Planejamento Estratégico, a gestão estratégica de preços, a precificação em modelos de receita recorrente, e o pricing dinâmico e a segmentação de clientes. Em seguida, a seção 3 aborda a metodologia utilizada, e a seção 4 os resultados obtidos. Finaliza-se o estudo na seção 5, a qual abrange a conclusão, limitação do tema e caminhos para pesquisas futuras.

\section{Referencial teórico}

\subsection{Planejamento estratégico}

O PE pode ser definido como o processo através do qual a empresa se mobiliza para atingir o sucesso e construir seu futuro, por meio de um comportamento pró-ativo, que considera seu ambiente atual e posterior (Pagnoncelli \& Vasconcellos, 1992). O PE delimita o caminho mais adequado a ser executado para alcançar objetivos e desafios da empresa (Oliveira, 1999) e se mostra como uma ferramenta gerencial valiosa (Bryson, Edwards \& Van Slyke, 2018).

Segundo Kotler \& Armstrong (2018), as empresas bem-sucedidas no mercado praticam frequentemente o PE. Dentre suas principais vantagens gerenciais, destacam-se a melhoria de controle e sistematização de ciclos de aperfeiçoamento organizacionais. Além disso, a consolidação de um PE traz agilidade e fundamenta decisões; ou seja, cria um consenso natural entre os líderes empresariais sobre o que é importante (Müller, 2003).

Diante de tamanha importância, O PE deve ser cuidadosamente estruturado, através de uma gestão que alinhe os recursos da organização e as oportunidades de mercado em permanente modificação (Kotler \& Armstrong, 2018). Segundo Müller (2003), os programas de melhoria sem a visão estratégica muitas vezes atacam problemas que não necessariamente possuem influência externa, ou seja, estes programas por vezes pecam por possuir uma visão exclusivamente interna. A figura 1 evidencia as fases necessárias para a condução de um PE consistente e traz os ambientes e delimitações necessários para sua análise. 
Figura 1

Condução do PE

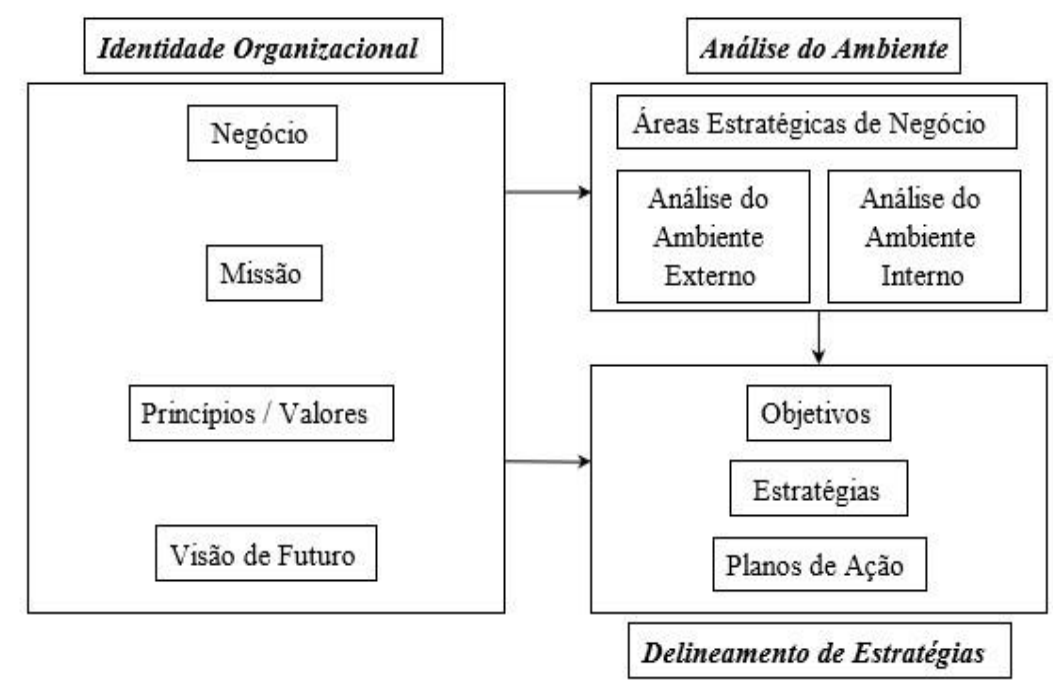

Fonte: Müller (2003).

Em suma, um PE adequado consiste na consolidação da Identidade Organizacional e na análise dos ambientes externos e internos da empresa. Apenas depois de definidos estes pontos, torna-se possível identificar e delimitar as estratégias e planos de ação que buscam atingir objetivos comuns da organização (Müller, 2003). É importante complementar que as áreas estratégicas e o ambiente interno de uma empresa podem vir a se alterar periodicamente, visto que dependem de fatores externos e de mercado, os quais estão em constante mutação (Kreiner et al., 2015). Dessa forma, é importante manter uma rotina de revisão e atualização do PE, de modo que a delimitação de estratégias sejam as mais assertivas possíveis.

Arend et al. (2015), ao discutir o papel do PE dentro das organizações, ressalta que ele é um processo complexo e que pode ser percebido pelos funcionários como facilitador ou coercitivo, dependendo das características da empresa. Por um lado, o PE apresentou um efeito positivo no desempenho financeiro, por outro, ele impactou negativamente a atividade inovadora das empresas. Assim, ao estabelecer um PE, os gestores precisam implementar incentivos de compartilhamento de conhecimento e capacitação dos funcionários para dirimir esses efeitos negativos (Arend et al., 2015).

\subsection{Gestão estratégica de preços}

A gestão de preços desempenha um papel importante nas estratégias de negócio de uma organização, especialmente no que tange à valorização e à manutenção do interesse dos clientes nos produtos ou serviços da empresa. Segundo Kotler e Armstrong (2018), os custos do produto definem o piso do preço a ser cobrado, e a percepção do consumidor quanto ao valor do produto definem o teto. Os autores destacam também que, por meio da determinação do preço, é possível desempenhar os 
objetivos que definem as estratégias, sendo necessário levar em consideração os preços concorrentes e outros fatores externos e internos à empresa.

Através da abordagem das três lentes do Pricing, que são valor para o cliente, concorrência e economia, é possível atingir o real valor do produto, quando se compreende o olhar através da intersecção das três lentes. Quando a empresa aplica a essa perspectiva, ela atinge uma vasta visão de possíveis oportunidades e uma garantia do melhor direcionamento do valor cobrado (BCG, 2014).

Determinar o quanto o consumidor está disposto a pagar por um produto ou serviço não é tarefa trivial, exigindo métodos bastante rigorosos de pesquisa mercadológica, que mesmo assim não garantirão completo sucesso (Dias, 2006). Assim, as três lentes esquematizadas pelo BCG (2014) visam a facilitar o entendimento do que deve ser mensurado para então tanger o valor que mais represente o produto ou serviço. As três lentes são (BCG, 2014):

- Valor para o cliente: o valor percebido pelo cliente não é algo fácil de ser mensurado (Kotler \& Armstrong, 2018). É necessário então olhar pela lente do cliente e se perguntar quem são cada um dos segmentos alvos e quanto estão dispostos a pagar pelo produto.

- Concorrência: aplicando a lente da competição é importante se perguntar quem são os concorrentes que oferecem alternativas e comparar o valor dos produtos oferecidos a partir de uma avaliação do produto ao longo do tempo.

- Economia: considerando que cada organização possui um conjunto exclusivo de considerações econômicas que influenciam suas decisões de compra, nessa lente é fundamental avaliar essas métricas específicas, como custo marginal, taxas de anexação e quedas de margem.

A literatura de gestão estratégica de preços está em grande parte focada na precificação de produtos e bens materiais, embora seja crescente o número de estudos focados também na precificação de serviços (Kienzler \& Kowalkowski, 2017). Kung e Zhong (2017), por exemplo, desenvolveram um modelo de precificação para plataformas de entrega (delivery) no qual os consumidores pagam uma assinatura para se tornarem membros, gerando um modelo de receita recorrente, o qual será abordado na próxima seção.

\subsection{Precificação em modelos de receita recorrente}

Um modelo de negócio com receita recorrente é, em geral, mais complexo que os modelos tradicionais (Cohen \& Neubert, 2019). Em vez de criar uma transação de receita única, no modelo recorrente, cada venda cria um fluxo de caixa contínuo. Esse fluxo dura enquanto o cliente permanecer 
inscrito no serviço/produto oferecido pela empresa, e é em geral monetizado com o pagamento de parcelas mensais, o que configura a lógica de assinatura (Harden, 2014).

Ao analisar um negócio baseado em receitas recorrentes é necessário, portanto, considerar suas diferenças quanto a modelos tradicionais de organização (Tabela 1). Essa teoria é confirmada por Harden (2014), que destaca as diferenças em todo o negócio, desde a maneira de vender, de fazer marketing, engajar clientes e medir o sucesso. Para o autor, não se está apenas vendendo um produto; em modelos de receita recorrente, a empresa precisa construir um relacionamento com o seu consumidor, pois a duração deste relacionamento determinará a continuidade da receita periódica.

\section{Tabela 1}

Diferenças entre modelos de receitas tradicionais recorrentes

\begin{tabular}{|c|c|}
\hline Modelo Tradicional & Modelo de Receita Recorrente \\
\hline Transacional & \begin{tabular}{c} 
Relacionamento é baseado em eventos \\
\hline Ordem/Pagamento é o ponto final \\
Ordem/Pagamento é o ponto inicial de um modelo \\
de aquisição, nutrição e \\
crescimento
\end{tabular} \\
\hline Nutrição \\
\hline Uma repetidas & \begin{tabular}{c} 
Reter e Renovar \\
\hline
\end{tabular} \\
\hline Upsell e cross-sell \\
\hline
\end{tabular}

Fonte: Harden (2014), adaptada pelos autores.

Para se obter sucesso em um modelo de assinaturas, é preciso trabalhar ao máximo em estratégias de redução de cancelamentos (Fruchter \& Sigué, 2013). Portanto, a empresa precisa entregar continuamente sua proposta de valor e, ao mesmo tempo, possuir uma estreita relação com seu cliente.

Um correto modelo de monetização pode acelerar a estratégia do negócio, ajudar no alcance de resultados e nas vantagens competitivas organizacionais. Em um modelo de receita recorrente, existe uma ampla variedade de oportunidades no que tange a serviços, necessidades do consumidor e dimensionamento de preços (Harden, 2014). Segundo Raddats, Burton, \& Ashman, (2015), a monetização dos serviços ofertados é um aspecto fundamental para que a organização atinja seus objetivos, devendo estar alinhada com os aspectos do PE.

Estudos em receita recorrente vêm ganhando bastante atenção da literatura devido à crescente oferta de softwares e hardwares como serviços (processo conhecido como servitização), pagos através de mensalidade (Cohen \& Neubert, 2019; Dempsey \& Kelliher, 2018), bem como pela oferta de variados serviços de entretenimento pagos por meio de mensalidade (Randall, Lewis, \& Davis, 2016). Entre as 
práticas para melhorar os negócios de receita recorrente estão a segmentação de Clientes e estratégias de pricing dinâmico, abordados na sequência.

\subsection{Segmentação de clientes}

Segundo Sibata (2017), os consumidores apresentam características diferentes entre si e, pela limitação de competências e recursos das empresas, torna-se impossível atender a todos os perfis de forma igualitária. Contudo, os clientes são essenciais para a saúde do negócio, e dessa forma, é importante que a empresa seja flexível para se adaptar a diferentes perfis (Ávila, 2014). A partir da segmentação de clientes, é possível aprofundar conhecimentos sobre eles, e desenvolver estratégias compatíveis a grupos específicos.

A segmentação é uma maneira prática e objetiva de aumentar a precisão de ações de marketing e precificação das empresas, ao identificar grandes grupos de consumidores por meio de características comuns - como poder de compra, preferências, localização, hábitos e atitudes (Hjort, Lantz, Ericsson, \& Gattorna, 2013). Segundo Ayerbe et al., (2014) e Raddats, Burton, \& Ashman (2015), é necessário reservar algum tempo para criar a segmentação de clientes. Esse processo, e a forma como é feito, pode resultar em impactos positivos na ativação e retenção de clientes, sob a perspectiva de negócios com receita recorrente. Estudos recentes aplicam ferramentas avançadas para realizar a segmentação de clientes, como método de cluster (Namvar, Gholamian, \& KhakAbi, 2010) e técnicas de data mining (Chen, Sain \& Guo, 2012).

\subsection{Estratégias de pricing dinâmico}

A estratégia de pricing dinâmico surgiu a partir de uma necessidade de adequar a precificação através da personalização do cliente, possibilitando capturar o maior valor possível de cada segmento de comprador (Montes \& Chaves 2017). Para Elmaghraby e Keskinocak (2003), a técnica de preços dinâmicos é utilizada há muito tempo em setores como companhias aéreas, hotéis e empresas de energia elétrica, em que a capacidade é fixada a curto prazo e é perecível. Complementarmente, nos últimos anos, houve uma crescente adoção de políticas dinâmicas de preços no varejo e em outros setores (Elmaghraby, \& Keskinocak 2003). No entanto, o marketing de massa introduziu mais ciência nesse processo: inicialmente com pesquisas demográficas e, mais recentemente, com as tecnologias de Big Data, Analytics e Machine Learning (Gupta \& Pathak, 2014). Dessa forma, essas tecnologias criam valor para as empresas, por meio da descoberta de padrões e relacionamentos entre dados.

Uma abordagem de precificação dinâmica é usada para tomar a decisão do preço de aquisição para os produtos usados com diferentes níveis de qualidade retornando das segmentações dos clientes 
(Keyvanshokooh, Fattahi, Seyed-Hosseini, \& Tavakkoli-Moghaddam, 2013). É possível seguir diferentes estratégias para compor um preço dinâmico, de acordo com o objetivo do negócio:

1- Preços segmentados: se refere a segmentação do preço para diferentes tipos de clientes prédefinidos (Abrate, Fraquelli, \& Viglia, 2012). Essa prática de preços reflete a lente de Pricing definido por BCG (2014) como "valor para o cliente".

2- Precificação de pico: é utilizado para elevar o valor cobrado em momentos de alta demanda (Faruqui \& Sergici, 2010).

3- Condições de mudança: usado para aumentar os lucros quando as condições do mercado estão mudando (DiMicco, Greenwald, \& Maes, 2001). Essa estratégia funciona através da diminuição do preço à medida que as vendas começam a cair e depois se aumenta o preço novamente.

De forma geral, é fundamental o estudo de características e comportamentos do consumidor, relacionando indicadores importantes para a empresa, como o preço, com a percepção de valor dos seus clientes, que podem variar. Conforme Kumar (2007), a personalização em massa é utilizada em estratégias com o foco em clientes para desenvolver pacotes de serviços e produtos satisfazendo as necessidades específicas de cada um deles. Aspira-se assim, a partir de um planejamento estratégico consistente, a estruturação de uma gestão de preços adaptável ao modelo de negócio da empresa e que compreenda as singularidades de seu público.

A precificação dinâmica vem sendo discutida pela literatura em diversos segmentos da economia. Bayoumi, Saleh, Atiya e Aziz (2012) criaram um algoritmo de otimização de receita que incorpora estratégias dinâmicas de preço para o segmento hoteleiro. Fruchter e Sigué (2013) construíram um modelo que apresenta e evolução da dinâmica de preços para serviços de assinatura. Já Jiaqi Xu, Fader e Veeraraghavan (2019) desenvolveram um modelo de precificação dinâmica para a venda de ingressos em jogos de baseball.

\section{Procedimentos metodológicos}

Quanto à sua natureza, este trabalho é classificado como uma pesquisa aplicada, caracterizando-se pela utilização de resultados na solução de problemas reais de uma empresa (Diehl \& Tatim, 2004). A pesquisa é definida como tendo uma abordagem qualitativa, por tratar em profundidade aspectos da empresa, e compreender mecanismos de soluções do problema, visto que, na área de gestão, a abordagem qualitativa é fundamental para que os pesquisadores possam lidar com a complexidade de temas e do contexto e com a multiplicidade de fatores e relacionamentos (Cassel \& Gummesson, 2006). Em relação aos seus objetivos, o presente artigo pode ser qualificado como 
pesquisa descritiva, pois busca descrever as características e os mecanismos de funcionamento dentro do ambiente organizacional proposto, coletando-se dados sobre os mais diversos aspectos (Cervo \& Bervian, 2011). Quanto aos procedimentos, a pesquisa é considerada uma observação participante (Taylor \& Bogdan, 1992), dado que os pesquisadores engajaram-se na situação estudada e conseguiram observar com profundidade a mesma; os participantes estavam em uma dupla condição (participantes e pesquisadores).

Para a realização deste trabalho, foi escolhida uma empresa brasileira do ramo de multimídias, com plataformas de televisão, rádio e jornal. Essa empresa foi escolhida por ser referência na área de comunicação e mídia digital, e-commerce segmentado e mobile, atuando em setores de alto crescimento e com modelos inovadores. O estudo foi realizado no segmento de jornais, especificamente na área de assinaturas, pois esse vem passando por transformações importantes que requerem uma mudança de estratégia.

O posicionamento empresarial em relação a estratégias de assinaturas de jornal vem sofrendo mudanças nos últimos anos. Este fato pode ser explicado pela tendência de queda no interesse dos consumidores em manter assinaturas de jornais e revistas (Seamans \& Zhu, 2014). Diante desse fenômeno, a empresa estudada vem apostando na ampliação do seu conteúdo para meios digitais (através do site e aplicativos), além do impresso, de maneira a criar valor e ajudar a manter e captar clientes.

Em suma, há um esforço coletivo para a ampliação e manutenção da carteira ativa de assinantes, tanto no meio digital quanto no físico. Com isso, o gerenciamento de preços é tido como elemento fundamental na busca dos objetivos empresariais, utilizado tanto como pivô da busca e retenção de assinantes, quanto como forte indicador da saúde do negócio.

Para a elaboração deste estudo, foi necessária a criação de uma sistemática metodológica, realizada a partir da união de elementos citados na seção 2 deste presente artigo. Primeiramente, realizou-se uma revisão do Planejamento Estratégico da organização, segundo a delimitação de Müller (2003). Com isso, buscou-se esclarecer os principais objetivos da organização e o que se espera para o futuro.

Em seguida, foi possível definir a estratégia de preços mais adequada àquele modelo de negócio - tanto em relação à gestão de preços quanto à prática mais apropriada do pricing dinâmico - visto que a precificação está diretamente relacionada à estrutura e ambiente em que a empresa está inserida. Posteriormente, realizou-se a segmentação de clientes, através da definição de diferentes perfis de consumidores. Para isso, houve a escolha detalhada de critérios e indicadores importantes para a análise do cliente, seguido da clusterização e classificação dos perfis em categorias diferentes, com base em características comuns. 
A partir da segmentação de clientes, designaram-se preços distintos para cada grupo, de acordo com as variáveis importantes ao objeto de estudo, ou seja, cada pessoa estava alocada em um grupo e associada a um valor para o mesmo produto. Em seguida, testou-se a eficiência das segmentações de preços por perfis de clientes através de testes. O primeiro experimento foi realizado através de uma ação de marketing já praticada anteriormente pela empresa. Após a realização do teste proposto foi possível medir a eficácia da ação e, por fim, discutir a viabilidade da implementação da estratégia em larga escala na empresa.

\section{Resultados e discussões}

Previamente à condução dos resultados deste estudo, cabe ressaltar que a empresa já utilizava uma tabela de preços para ofertar valores promocionais na assinatura dos clientes, na qual considerava fatores como custo e margem de lucro. Os valores ofertados se baseavam exclusivamente pelo ano da tabela praticada, ou seja, pelo histórico de preço mensal das modalidades de acordo com o produto assinatura de jornal impresso ou de jornal digital - que são reajustados anualmente. As modalidades de assinatura de jornal físico são nomeadas pelo total de dias da semana que se recebe o jornal ( 6 dias, 5 dias, 3 dias, por exemplo), considerando um máximo de seis jornais por semana, pois sábado e domingo são contemplados em uma única edição. Já no jornal digital, as modalidades são nomeadas de acordo com as plataformas de acesso assinadas, podendo ser apenas o site e o aplicativo de notícias (básica) ou incluindo o aplicativo do jornal digital (completa).

A representação da tabela de preços mensais históricos está exibida na Tabela 2, com valores mascarados para preservar o sigilo organizacional. É ainda considerado o reajuste que se mantém constante a cada ano nas modalidades de jornais impresso. Já nas modalidades de jornal digital, o preço mensal histórico, também fictício, foi representado com apenas um ajuste de aumento de preço no ano de 2014.

\section{Tabela 2}

Precificação previamente estipulada para as modalidades principais de cada produto

\begin{tabular}{|c|c|c|c|c|c|c|}
\hline \multirow{2}{*}{ Ano } & \multicolumn{4}{|c|}{ Jornal Físico } & \multicolumn{2}{|c|}{ Jornal Digital } \\
\hline & 6 dias & 5 dias & 3 dias & 2 dias & Completo & Básico \\
\hline 2018 & $R \$ 190,00$ & $\mathrm{R} \$ 149,00$ & $\mathrm{R} \$ 91,00$ & $R \$ 68,00$ & $R \$ 35,00$ & $R \$ 20,00$ \\
\hline 2017 & $\mathrm{R} \$ 185,00$ & $R \$ 146,00$ & $\mathrm{R} \$ 89,00$ & $R \$ 67,00$ & $\mathrm{R} \$ 35,00$ & $R \$ 20,00$ \\
\hline 2016 & $\mathrm{R} \$ 180,00$ & $R \$ 143,00$ & $\mathrm{R} \$ 87,00$ & $R \$ 66,00$ & $R \$ 35,00$ & $R \$ 20,00$ \\
\hline 2015 & $\mathrm{R} \$ 175,00$ & $\mathrm{R} \$ 140,00$ & $\mathrm{R} \$ 85,00$ & $R \$ 65,00$ & $\mathrm{R} \$ 35,00$ & $R \$ 20,00$ \\
\hline 2014 & $R \$ 170,00$ & $R \$ 137,00$ & $R \$ 83,00$ & $R \$ 64,00$ & $R \$ 30,00$ & $R \$ 15,00$ \\
\hline 2013 & $R \$ 165,00$ & $\mathrm{R} \$ 134,00$ & $\mathrm{R} \$ 81,00$ & $R \$ 63,00$ & $\mathrm{R} \$ 30,00$ & $\mathrm{R} \$ 15,00$ \\
\hline 2012 & $R \$ 160,00$ & $\mathrm{R} \$ 131,00$ & $\mathrm{R} \$ 79,00$ & $R \$ 62,00$ & $\mathrm{R} \$ 30,00$ & $R \$ 15,00$ \\
\hline 2011 & $R \$ 155,00$ & $\mathrm{R} \$ 128,00$ & $R \$ 77,00$ & $R \$ 61,00$ & $R \$ 30,00$ & $R \$ 15,00$ \\
\hline 2010 & $R \$ 150,00$ & $\mathrm{R} \$ 125,00$ & $\mathrm{R} \$ 75,00$ & $R \$ 60,00$ & - & - \\
\hline
\end{tabular}


O preço praticado em cada modalidade é proporcional ao custo envolvido em cada produto (custo de entrega, matéria-prima e logística) e a margem de lucro esperada pela empresa. Além disso, as margens de descontos para cada produto e modalidade já estavam orçadas previamente à realização deste estudo e denominados pelo valor de tabela anual (de 2010 a 2018). Dessa forma, conforme evidenciado na Tabela 2, o preço máximo (correspondente ao último reajuste) o qual um assinante pode pagar pela sua modalidade é a Tabela 2018, enquanto que o preço mínimo (aquele com o maior percentual de desconto) é a Tabela 2010 para o jornal físico e a Tabela 2011 para o jornal digital.

O motivo pelo qual se estruturou esse modelo de tabelas anuais com maiores e menores preços por modalidades é devido à possibilidade de a empresa ter poder de barganha com seus clientes, e negociar valores até o limite de sua margem de lucro. A tabela mínima é, em teoria, utilizada apenas para evitar o cancelamento de um assinante que alega problema financeiro ou falhas graves de entrega, de modo que sua utilização não é incentivada.

\subsection{Revisão do planejamento estratégico}

A revisão do Planejamento Estratégico foi realizada a partir do esquema desenvolvido por Müller (2003) e está representada na Figura 2. Em relação à Identidade Organizacional, cabe ressaltar que o setor a ser estudado será o de assinaturas de jornais. Seus princípios e valores, como desenvolvimento e a relação com o cliente, evidenciam a importância da perspectiva dos consumidores.

Figura 2

Revisão do PE

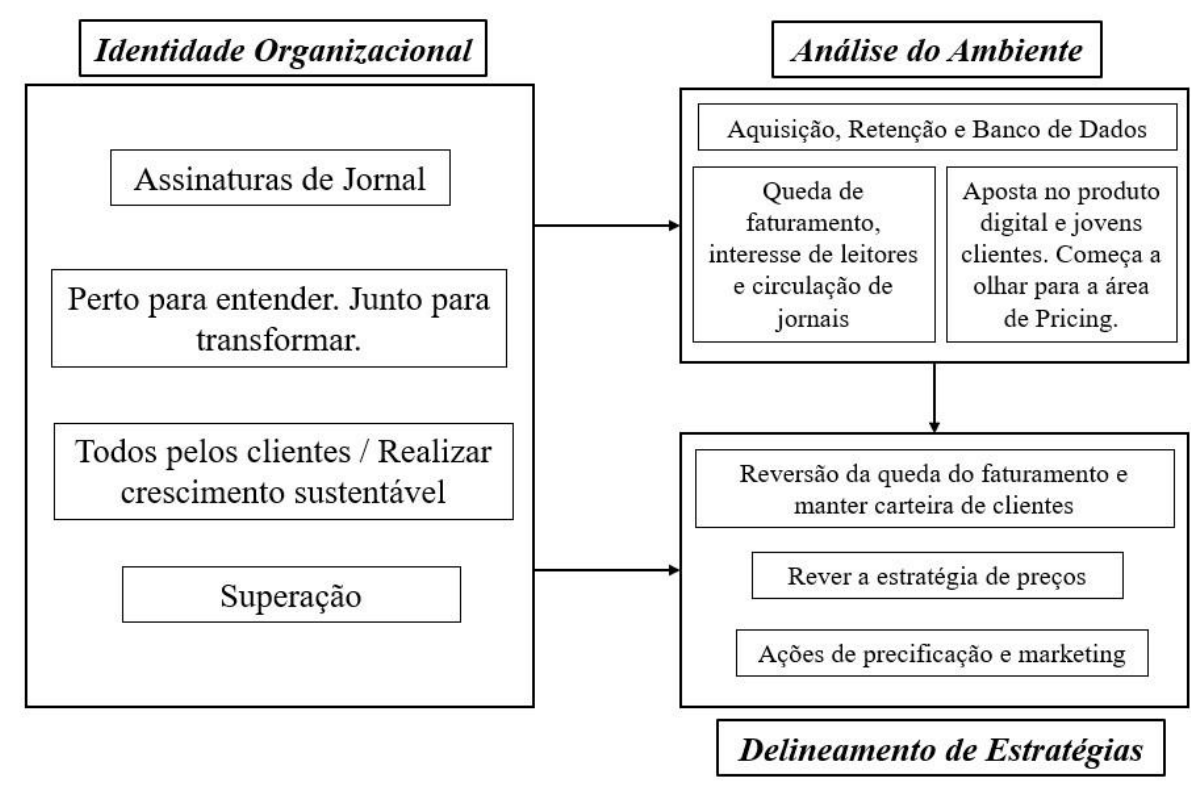

Fonte: Elaborado pelos autores. 
A respeito da Análise do Ambiente, percebe-se que a empresa vivencia um momento delicado em relação ao mercado externo, visto que há queda de faturamento e comercialização de jornais impressos. Com isso, evidencia-se a necessidade de adequar o ambiente interno da organização para tal situação, o que ocorre pela aposta em seu produto digital.

No Delineamento de Estratégias, há o reflexo da Identidade Organizacional e do Ambiente da empresa. Logo, como macro objetivo, está a reversão da queda de faturamento e a manutenção da carteira de assinantes do produto oferecido, seja jornal impresso ou digital. Em relação às estratégias, define-se a revisão do preço, de forma que seja mais assertivo ao público destinado e diminua os cancelamentos de assinaturas por motivo de valor. Como plano de ação, é possível utilizar ferramentas de marketing da empresa, o que possibilita sua prática mensuração.

\subsection{Definição da estratégia de preços}

Após a análise minuciosa do Planejamento Estratégico da empresa, conforme sugerido por Müller (2003), notou-se que o estudo e a redefinição das práticas em políticas de preço eram adequados frente ao contexto da empresa, tanto em questões de identidade quanto em relação aos ambientes interno e externo. Assim, um programa de melhoria de gestão de preços atacaria um problema real da empresa e, por fim, traria vantagens competitivas para a organização.

Diante das teorias consolidadas na literatura, foram escolhidas as direções mais apropriadas para a execução do estudo na empresa em questão. O modelo de negócio baseado em assinaturas, cujo fluxo de receita é recorrente, ressalta a importância dos clientes e a sua relação com o produto. Por esse motivo, dentro das três esferas de pricing, propostos por BCG (2014), para a concretização do valor do produto, optou-se por dar foco ao segmento de "valor para o cliente". Ademais, por tratar-se de uma empresa de grande porte, há inúmeros perfis de clientes e modalidades de produtos, o que propiciou a definição de "preços segmentados", uma das estratégias de Pricing Dinâmico. Dessa forma, a precificação dinâmica e clusterizada foi definida pelos autores como estratégia de preços mais adequada para se seguir diante da conjuntura organizacional.

\subsection{Segmentação de clientes}

Para a segmentação de clientes, utilizou-se o banco de dados da empresa e variáveis que se mostraram importantes, como o valor pago, o tempo de assinatura, modalidades do produto utilizadas, entre outros. Assim, diferentes perfis de assinantes puderam ser criados e então organizados, conforme segue. Um assinante de jornal impresso, a partir de variáveis como valor pago (podendo ter ou não desconto na mensalidade), histórico de contato com o Call Center (número de vezes que ligou para reclamar ou foi contatado para cobrança de parcela não paga) e tempo de assinatura, foi classificado 
entre "Antigo", "Vip" e "Novo". Já os assinantes de jornal digital, foram divididos entre "Antigo", "Digital/Call Center" e "Digital", de acordo com a forma que se relacionaram com a empresa para adquirir sua assinatura (pelo site ou pelo Call Center) e pelo tempo de carteira.

Por fim, ao segregar a base de dados dos assinantes a partir dos critérios de avaliação, foi possível realizar uma análise de comportamento dos seis perfis individuais, considerando suas particularidades. Esses indicadores abertos para cada perfil, exibidos no Tabela 3, foram considerados primordiais para o estudo da vida do assinante no modelo de receita recorrente da empresa. As variáveis mostram: o nome do perfil/cluster; o ticket médio, ou seja, a média do valor mensal pago por esse grupo de assinantes; a porcentagem da base total de assinantes que esse perfil representa; o percentual de homens no grupo; a média de idade dos assinantes; o uso do produto digital - site ou aplicativos - nos últimos três meses; e a modalidade com maior representatividade no cluster. Cabe ressaltar que todos os assinantes do jornal (seja ele impresso ou digital) têm direito ao acesso no site e no aplicativo da empresa.

\section{Tabela 3}

Abertura das variáveis de cada perfil de assinante

\begin{tabular}{|c|c|c|c|c|}
\hline Produto/Critérios & Perfil & Ticket Médio & $\%$ Base & Gênero Masculino \\
\hline \multirow{3}{*}{ Digital } & Antigo & $R \$ 20,00$ & $10 \%$ & $75 \%$ \\
\hline & Dig/Call & $\mathrm{R} \$ 30,00$ & $10 \%$ & $55 \%$ \\
\hline & Digital & $\mathrm{R} \$ 25,00$ & $5 \%$ & $60 \%$ \\
\hline \multirow{3}{*}{ Impresso } & Antigo & $R \$ 125,00$ & $40 \%$ & $45 \%$ \\
\hline & Vip & $R \$ 175,00$ & $30 \%$ & $50 \%$ \\
\hline & Novo & $R \$ 110,00$ & $5 \%$ & $45 \%$ \\
\hline \multirow[t]{2}{*}{ Produto/ Critérios } & Média idade (anos) & Média tempo base & $\begin{array}{l}\text { Média uso digital } \\
\text { últimos } 3 \text { meses }\end{array}$ & $\begin{array}{c}\text { Modalidade } \\
\text { Principal }\end{array}$ \\
\hline & 45 & 8 meses & $90 \%$ & 85\% Básica \\
\hline \multirow[t]{3}{*}{ Digital } & 55 & 5 anos & $65 \%$ & $80 \%$ Completa \\
\hline & 50 & 5 meses & $70 \%$ & 70\% Completa \\
\hline & 60 & 10 anos & $25 \%$ & $55 \% 6$ dias \\
\hline \multirow[t]{2}{*}{ Impresso } & 65 & 15 anos & $20 \%$ & $70 \% 6$ dias \\
\hline & 55 & 5 meses & $35 \%$ & $65 \% 6$ dias \\
\hline
\end{tabular}

Fonte: Elaborado pelos autores.

A partir dos resultados iniciais mostrados no Tabela 3, torna-se claro que há diferenças relevantes entre os clusters de clientes criados. Portanto, evidencia-se a necessidade de atuar sobre cada segmento de forma individual, considerando as singularidades de cada um, principalmente ao colocar em prática as estratégias organizacionais. Desse modo, é possível estruturar diferentes planos de ação para os assinantes (e ex-assinantes) do jornal, desde sua fidelização com a marca, campanhas de marketing e relacionamento, e diminuição do churn de assinaturas (taxa de cancelamento). 
Sendo o percentual de cancelamentos mensais de assinatura um dos indicadores mais relevantes em uma empresa com modelo de receita recorrente, é indispensável que sejam estabelecidas estratégias que auxiliem a diminuição dessa taxa. Logo, a separação dos assinantes em categorias, conforme critérios anteriormente discutidos, serve também como facilitador para a delimitação de preços dinâmicos e personalizados.

Dessa forma, para cada grupo de perfil de cliente haverá uma tabela de preço específica (conforme a Tabela 4), de forma que auxilie na retenção desses clientes com intenção de cancelamento no Call Center da empresa e sirva como apoio de argumentação do operador no momento de recuperar aqueles assinantes que cancelaram há pouco tempo.

\section{Tabela 4}

Exemplo de segmentação da oferta por perfil - considerando apenas o produto impresso

$\begin{array}{cccc} & \text { Cluster 1 } & \text { Cluster 2 } & \text { Cluster 3 } \\ \text { Perfil } & \text { Antigo } & \text { Vip } & \text { Novo } \\ & \text { Assinaram por mais de } & \text { Nunca obtiveram desconto e } & \text { Clientes com menores } \\ \text { Considerações } & \text { dois anos e representam } & \text { nunca ligaram para o Call } & \text { preços e que assinaram por } \\ & \text { quase metade da carteira } & \text { Center; perfil que prefere mais } & \text { pouco tempo } \\ \text { da empresa } & \text { jornal/semana } & \\ \text { Ofertas } & \text { Assinatura de } 5 \text { ou 3 dias } & \text { Assinatura de 6 ou 5 dias por } & \text { Assinatura de 3 ou 2 dias } \\ & \text { por valores de 2014 } & \text { valores de 2015 } & \text { por valores de 2012 }\end{array}$

Fonte: Elaborado pelos autores

A tabela 4 traz a nova organização de preços propostos para a empresa, exemplificados por meio dos perfis de jornal impresso (Antigo, Vip e Novo). A partir dessa visão, é possível perceber que a designação de valores e descontos passa a seguir uma estrutura padronizada e lógica. Assim, aqueles assinantes do jornal físico que estão habituados a procurar descontos, mas ao mesmo tempo apreciam o produto diário - clientes Antigos - poderão ser migrados para um preço de tabela 2014. Já para aqueles assinantes com perfil Vip, que costumam pagar o preço cheio e enxergam maior valor no jornal, deve ser concedido o desconto da tabela 2015. Os clientes Novos, os quais não possuem forte aproximação com o produto e, consequentemente, buscam pagar menos, podem adquirir sua assinatura pela tabela de 2012.

\subsection{Aplicação dos preços segmentados}

Após a alocação de descontos específicos para cada perfil de assinante, procurou-se experimentar a efetividade de tal segmentação. Para isso, foi realizada uma ação de envio de SMS com ofertas determinadas para cada cluster criado. O público a ser alcançado era o de ex-assinantes, ou seja, 
os valores eram ofertados com o objetivo de que esses clientes voltassem a possuir o jornal. Como o envio de mensagens via SMS pela empresa era uma ferramenta comum e, anteriormente utilizada, foi possível a mensuração de resultados tangíveis em relação à segmentação de preços.

O resultado da ação de SMS mostrou-se positivo no primeiro teste realizado. A conversão, ou seja, o total de (ex) clientes que aceitaram a oferta e voltaram a ser assinantes do jornal foi $100 \%$ maior do que a média obtida em tentativas anteriores, em que não se praticava a segmentação de clientes com envio de ofertas personalizadas por perfil. Assim, constatou-se a possibilidade de, por meio de ações fundamentadas em pricing dinâmico segmentado, melhorar as recuperações de clientes e enriquecer o relacionamento entre a empresa e o assinante.

Com base nas conversões obtidas através do primeiro experimento de uma estratégia de pricing dinâmico, procurou-se expandir a sua utilização dentro da empresa estudada. Dessa forma, elaboraramse análises mais aprofundadas em relação aos perfis da carteira de assinantes, assim como a sua aplicação em cenários distintos.

\subsection{Sugestão de aplicação futura}

A partir dos resultados citados anteriormente, foi estruturado um novo modelo para a retenção de assinantes com intenção de cancelamento no Call Center. O objetivo é delimitar os descontos oferecidos aos clientes de acordo com as segmentações definidas no item 4.4 deste artigo, de forma que fortaleça e padronize a política de preços praticadas. A aplicabilidade do modelo é dependente de ajustes de TI no Sistema de Informação da empresa, para que o dado a respeito do perfil do assinante esteja claro e visível para todos os operadores de telemarketing de forma instantânea.

Com o acesso ao tipo de cluster que o cliente está classificado previamente, é possível fortalecer ainda mais a argumentação dos atendentes e a oferta de uma proposta que tenha mais propensão a ser aprovada. Dessa forma, os scripts e pontos de argumentação poderão ser treinados e estudados de acordo com cada assinante e sua trajetória até o momento, além ser possível oferecer preços mais personalizados, organizados e consistentes, ou seja, que não abram espaço para a perda de margem para a empresa.

A Figura 3 traz um esquema como exemplo de uma operação mantida no Call Center aliada a estratégia de preço segmentados. O operador saberá, no momento em que obtém acesso às informações mínimas do cliente no sistema, quais modalidades oferecer para os assinantes que entrarem em contato, ou seja, influenciará na sua estratégia de negociação com o assinante, flexibilizando o preço pago ou trocando a modalidade para uma com menor propensão a cancelar, por exemplo.

Figura 3 
Proposta para a segmentação de preços no Call Center

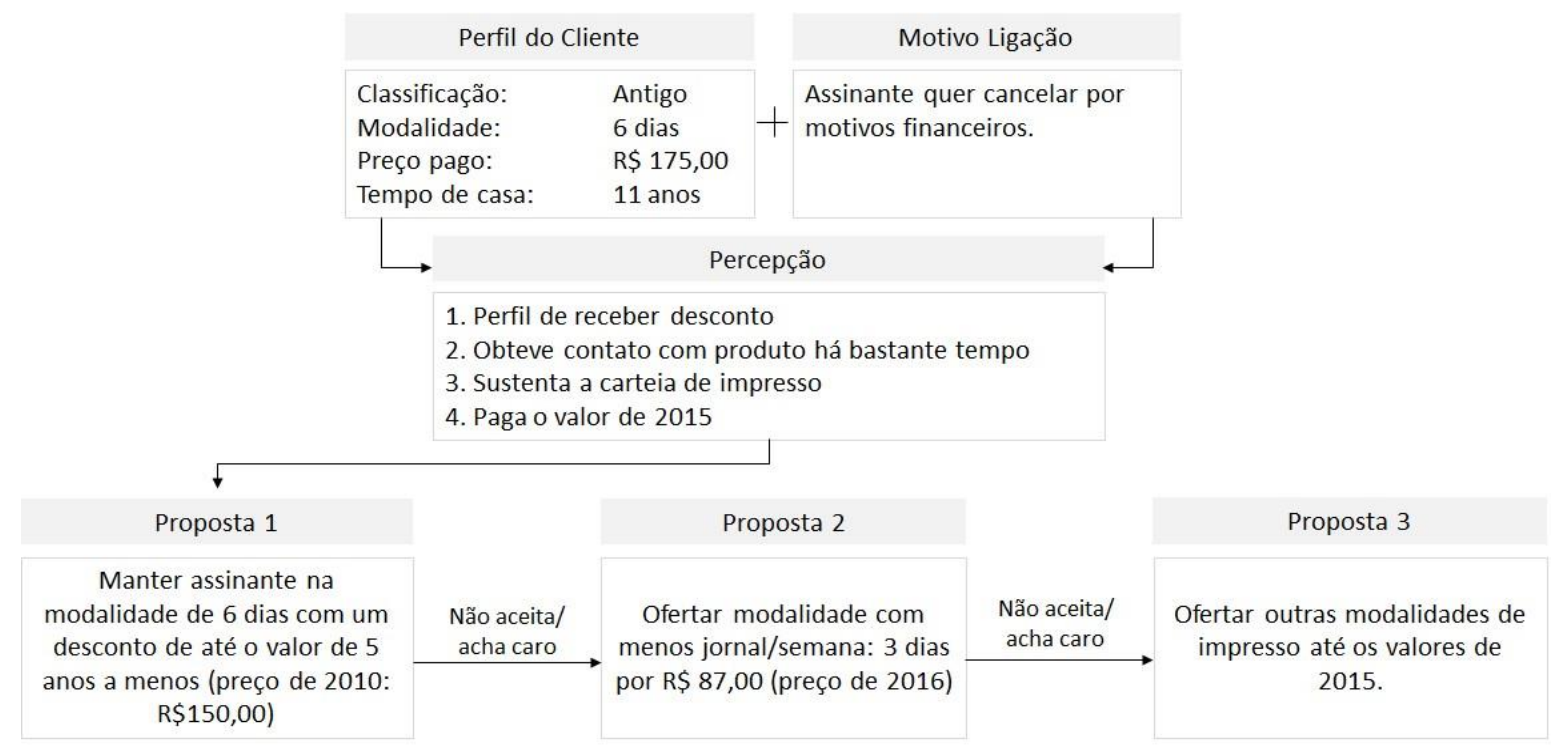

Fonte: Elaborada pelos autores

\subsection{Discussão}

O estudo iniciou com a revisita do Planejamento Estratégico da organização, uma vez que é imprescindível entender como funciona a identidade organizacional com intuito de entender tanto a dinâmica organizacional quanto a orientação de negócios e estratégia da empresa (Anthony \& Tripsas, 2016). A situação atual, de queda na venda de jornais impressos, mostrou a importância de se revisar o Ambiente Interno da organização; conforme Kreiner et al. (2015) a Identidade Organizacional é renegociada entre os membros ativos da organização, logo ela não é estática. Verificou-se que a redefinição da estratégia de precificação estava alinhada com o Planejamento Estratégico da empresa.

Os resultados descritos até então se mostraram satisfatórios diante das expectativas da abordagem de preços dinâmicos e segmentados. A estratégia de preços segmentados se mostra adequando quando a empresa possui um número razoável de diferentes clientes (Abrate, Fraquelli, \& Viglia, 2012). Dessa forma, é possível perceber as potenciais vantagens que a instauração de uma política de preços com tais características trariam para a organização, principalmente quanto à personalização e à percepção de valor do produto. A precificação dinâmica baseada em cluster tem sido utilizada recorrentemente na literatura e se mostrado como uma ferramenta importante para as empresas de diversos segmentos (Mahmoudi-Kohan, Moghaddam \& Sheikh-El-Eslami, 2010; Namvar, Gholamian, \& KhakAbi, 2010).

A partir das descobertas obtidas por meio deste estudo, é possível constatar informações importantes a respeito da empresa e de sua base de assinantes. Em relação aos clientes de jornal físico, nota-se que, quanto maior for o seu tempo de casa, ou seja, quanto mais antiga for a data da assinatura, maior será a sua percepção de valor quanto ao produto consumido. Além disso, esse segmento, 
classificado como Cliente Vip, tendem a possuir a média de idade mais elevada e, ao mesmo tempo, não costumam utilizar o site ou os aplicativos para ler as notícias.

Os assinantes classificados como "Antigos", do jornal impresso, também devem tornar-se um ponto de atenção para a empresa. Sua representatividade na carteira total é significativa, mas tendem a procurar pagar valores mais baixos. Por isso, muitos acabam por migrar para modalidades digitais e, posteriormente podem vir a cancelar a assinatura por não desenvolverem o hábito da leitura digital. Portanto, é preferível que a migração desse segmento de clientes ocorra sempre para uma modalidade semelhante com a de origem, de forma que o churn (taxa de cancelamento) não aumente. Para permanecer competitivo e lucrativo nesse segmento, é necessário limitar ativamente o atrito e desgaste do cliente (Fruchter \& Sigué, 2013), a fim de evitar seu cancelamento.

Em relação aos clientes do produto digital, que vêm expandido significativamente nos últimos anos, percebe-se uma relação muito mais instável do que a observada nos consumidores de produtos impresso. Uma justificativa para tal ação é a alta oferta de websites e blogs que oferecem notícias sem custo, principalmente porque o custo de reprodução da notícia é quase nulo, enquanto que o custo da produção original é alto (Brandtweiner, 2000). Mesmo o valor do produto sendo consideravelmente menor, o consumidor possui uma tendência mais alta de perder o interesse ou considerar que não vale o custo-benefício de sua assinatura, tendo em vista a análise dos seus percentuais de uso.

\section{Conclusão}

A partir de estudos, análises e aplicações, tornou-se possível a confirmação de que um gerenciamento de preços estruturados, além de trazer maior confiabilidade ao processo, auxilia na assertividade de ações e na geração de melhores resultados. Além disso, para garantir a abrangência e o alinhamento de todos os aspectos importantes para a empresa e o mercado no qual ela está inserida, a imersão no PE e no modelo de negócio da organização são necessários.

Sendo assim, o método de segmentação contribui não somente para ajustes de preço, mas também para o fortalecimento do conhecimento da empresa sobre seus clientes e a personalização de atendimentos e ofertas. Os seis segmentos resultantes da análise de clusters mostraram-se representativos, afinal, através das validações qualitativas e variáveis encontradas foi possível realizar um tratamento diferenciado e aumentar as conversões de assinaturas. A análise dos perfis pode, ainda, auxiliar os tomadores de decisão na definição de investimentos e cuidados para cada grupo separadamente, de forma a expandir a assertividade das ações da empresa em relação ao seu planejamento estratégico.

Por fim, conclui-se que a estratégia de pricing dinâmico segmentado pode ser ampliada para mais áreas da empresa, como o exemplo do Call Center e a implementação propriamente dita de ações 
estratégicas. Ademais, o uso das informações dos clientes enriquece a comunicação e indica o que exige mais atenção ou que deve ser tratado com prioridade. Além disso, esta análise pode auxiliar os pesquisadores a entenderem que pricing dinâmico, segmentação de clientes e geração de receita são pontos de partida para novos estudos.

Para a empresa, o estudo atinge o proposto em identificação dos segmentos, reformulação da organização de preços e proposta de melhorias. Conforme visto nos resultados, o pricing segmentado e adequado para cada perfil de cliente pode elevar o percentual de conversão em assinantes. Além disso, o estudo em si levanta novas questões a serem avaliadas, como, por exemplo, um maior número de ações de marketing para consolidar a escolha dos preços segmentados e a atualização constante dos perfis encontrados.

Apesar do escopo estar limitado à retenção de clientes e à geração de novas fontes receitas, por se tratar de um modelo de assinaturas, os resultados obtidos podem ser empregados em áreas como aquisição de clientes, análise do comportamento do consumidor e desenvolvimento de produto. Para pesquisas futuras, no próprio âmbito da clusterização, o modelo pode ser evoluído, melhorando ainda mais a robustez e fornecendo mais análises, uma vez que é necessário a execução de um maior número de testes com a clusterização de assinantes, para apurar a eficácia de tal prática. Além disso, diversos tomadores de decisão podem aplicar o estudo nas suas áreas para que consigam atingir seus objetivos estratégicos.

\section{Referências}

Abrate, G., Fraquelli, G., \& Viglia, G. (2012). Dynamic pricing strategies: Evidence from European hotels. International Journal of Hospitality Management, 31(1), 160-168. https://doi.org/10.1016/j.ijhm.2011.06.003

Anthony, C., \& Tripsas, M. (2016). Organizational identity and innovation. The Oxford Handbook of organizational identity, 417-435.

Arend, R. J., Zhao, Y. L., Song, M., \& Im, S. (2017). Strategic planning as a complex and enabling managerial tool. Strategic Management Journal, 38(8), 1741-1752. https://doi.org/10.1002/smj.2420

Ayerbe, A., Cirion, I., Torres, A. D., Gil, G., \& Laka, J. (2014, November). Thinking products in a different way: What is needed for product servitization. In 3rd International Business Servitization Conference Servitization (pp. 13-14). https://doi.org/10.3926/serv2014

Bayoumi, A. E.-M., Saleh, M., Atiya, A. F., \& Aziz, H. A. (2012). Dynamic pricing for hotel revenue management using price multipliers. Journal of Revenue and Pricing Management, 12(3), 271285. https://doi.org/10.1057/rpm.2012.44

BCG (2014). The Go-to-Market Revolution: A Growth Zealot's Guide to Commercial Transformation. Boston: The Boston Consulting Group, Inc. 
Brandtweiner, R. (2000). Pricing for Digitized Information Goods: The Case of Austrian Online Newspapers. AMCIS 2000. Proceedings. 284. http://aisel.aisnet.org/amcis2000/284

Bryson, J. M., Edwards, L. H., \& Van Slyke, D. M. (2018). Getting strategic about strategic planning research. Public Management Review, 20(3), 317-339. https://doi.org/10.1080/14719037.2017.1285111

Cassell, C., \& Gummesson, E. (2006). Qualitative research in management: addressing complexity, context and persona. Management Decision, 44(2), 167-179. https://doi.org/10.1108/00251740610650175

Cervo, A. L., \& Bervian, P. A. (2011). Metodologia científica: para uso dos estudantes universitários. In Metodologia científica: para uso dos estudantes universitários (pp. 144-144).

Chen, D., Sain, S. L., \& Guo, K. (2012). Data mining for the online retail industry: A case study of RFM model-based customer segmentation using data mining. Journal of Database Marketing \& Customer Strategy Management, 19(3), 197-208. https://doi.org/10.1057/dbm.2012.17

Cohen, M. C., Lobel, I., \& Paes Leme, R. (2020). Feature-based dynamic pricing. Management Science, 66(11), 4921-5484. https://doi.org/10.1287/mnsc.2019.3485

Cohen, B., \& Neubert, M. (2019). The influence of pricing strategies on corporate valuation. International Journal of Teaching and Case Studies, 10(2), 125-156. http://dx.doi.org/10.1504/IJTCS.2019.101503

Dempsey, D., \& Kelliher, F. (2018). Recurring Revenue Model Through a Cloud Computing Channel. Industry Trends in Cloud Computing, 111-128. http://dx.doi.org/10.1007/978-3-319-639949_6

Dias, S. R. (2006). Gestão de marketing. São Paulo: Saraiva.

Diehl, A. A., \& Tatim, D. C. (2004). Pesquisa em ciências sociais aplicadas: métodos e técnicas. Pearson Brasil.

DiMicco, J. M., Greenwald, A., \& Maes, P. (2001). Dynamic pricing strategies under a finite time horizon. IN Proceedings of the 3rd ACM Conference on Electronic Commerce - EC'01. https://doi.org/10.1145/501158.501169

Elmaghraby, W., \& Keskinocak, P. (2003). Dynamic pricing in the presence of inventory considerations: Research overview, current practices, and future directions. Management science, 49(10), 1287-1309. https://doi.org/10.1287/mnsc.49.10.1287.17315

Faruqui, A., \& Sergici, S. (2010). Household response to dynamic pricing of electricity: a survey of 15 experiments. Journal of Regulatory Economics, 38(2), 193-225. https://doi.org/10.1007/s11149-010-9127-y

Fruchter, G. E., \& Sigué, S. P. (2013). Dynamic pricing for subscription services. Journal of Economic Dynamics and Control, 37(11), 2180-2194. https://doi.org/10.1016/j.jedc.2013.05.003

Gupta, R., \& Pathak, C. (2014). A machine learning framework for predicting purchase by online customers based on dynamic pricing. Procedia Computer Science, 36, 599-605. https://doi.org/10.1016/j.procs.2014.09.060

Harden, B. (2014). The Guide to Recurring Revenue Success. São Francisco: Aria. 
Hjort, K., Lantz, B., Ericsson, D., \& Gattorna, J. (2013). Customer segmentation based on buying and returning behaviour. International Journal of Physical Distribution \& Logistics Management, 43(10), 852-865. https://doi.org/10.1108/ijpdlm-02-2013-0020

Jiaqi Xu, J., Fader, P. S., \& Veeraraghavan, S. (2019). Designing and Evaluating Dynamic Pricing Policies for Major League Baseball Tickets. Manufacturing \& Service Operations Management, 1-18. https://doi.org/10.1287/msom.2018.0760

Karlsen, S. S., Hamdy, M., \& Attia, S. (2020). Methodology to assess business models of dynamic pricing tariffs in all-electric houses. Energy and Buildings, 207, 109586.

https://doi.org/10.1016/j.enbuild.2019.109586

Keyvanshokooh, E., Fattahi, M., Seyed-Hosseini, S. M., \& Tavakkoli-Moghaddam, R. (2013). A dynamic pricing approach for returned products in integrated forward/reverse logistics network design. Applied Mathematical Modelling, 37(24), 10182-10202. https://doi.org/10.1016/j.apm.2013.05.042

Kienzler, M., \& Kowalkowski, C. (2017). Pricing strategy: A review of 22 years of marketing research. Journal of Business Research, 78, 101-110. https://doi.org/doi:10.1016/j.jbusres.2017.05.005

Kotler, P., \& Armstrong, G. (2018). Principles of Marketing (17.ed.). Harlow: Pearson Education Limited.

Kreiner, G. E., Hollensbe, E., Sheep, M. L., Smith, B. R., \& Kataria, N. (2015). Elasticity and the dialectic tensions of organizational identity: How can we hold together while we are pulling apart? Academy of Management Journal, 58(4), 981-1011.

https://doi.org/10.5465/amj.2012.0462

Kumar, A. (2007). From mass customization to mass personalization: a strategic transformation. International Journal of Flexible Manufacturing Systems, 19(4), 533.

Kung, L.-C., \& Zhong, G.-Y. (2017). The optimal pricing strategy for two-sided platform delivery in the sharing economy. Transportation Research Part E: Logistics and Transportation Review, 101, 1-12. https://doi.org/10.1016/j.tre.2017.02.003

Mahmoudi-Kohan, N., Moghaddam, M. P., \& Sheikh-El-Eslami, M. K. (2010). An annual framework for clustering-based pricing for an electricity retailer. Electric Power Systems Research, 80(9), 1042-1048. https://doi.org/10.1509/jimk.11.1.47.20136

Montes, H., \& Chaves, M. (2017). O Verdadeiro Valor do Pricing: Da estratégia de preços à excelência comercial. 2017. Deloitte Touche Tohmatsu.

https://www2.deloitte.com/content/dam/Deloitte/br/Documents/strategy/verdadeiro-valorpricing-tax.pdf

Müller, C.J. (2003). Modelo de gestão integrando planejamento estratégico, sistemas de avaliação de desempenho e gerenciamento de processos (MEIO - Modelo de Estratégia, Indicadores e Operações). Tese de doutorado. Universidade Federal do Rio Grande do Sul - UFRGS, Programa de Pós-Graduação em Engenharia de Produção - PPGEP. Porto Alegre. 
Namvar, M., Gholamian, M. R., \& KhakAbi, S. (2010). A two phase clustering method for intelligent customer segmentation. In 2010 International Conference on Intelligent Systems, Modelling and Simulation (pp. 215-219). IEEE. https://doi.org/10.1109/ISMS.2010.48

Oliveira, D. P. R. (1999). Excelência na administração estratégica: a competitividade para administrar o futuro das empresas (4 ed.). São Paulo: Atlas.

Pagnoncelli, D., \& Vasconcellos Filho, P. (1992). Sucesso empresarial planejado. Rio de Janeiro: Qualitymark.

Raddats, C., Burton, J., \& Ashman, R. (2015). Resource configurations for services success in manufacturing companies. Journal of Service Management. https://doi.org/10.1108/JOSM-122012-0278

Ramos, P. M., Maya, P. C. C., \& Bornia A. C. (2005). Um Estudo Científico do Componente Preço e sua Relação com o Marketing Mix de Produto Brasileiro de Exportação: uma Pesquisa Multicasos nas Empresas do Consórcio de Exportação de Calçados de São João Batista. In: ENCONTRO DA ANPAD, 29, 2005, Curitiba. Anais... Brasília: Anpad. http://www.anpad.org.br/diversos/down_zips/9/enanpad2005-mktc-1151.pdf

Randall, C., Lewis, A., \& Davis, A. (2016). How subscriptions are creating winners and losers in retail. Harvard Business Review, 1-6. https://hbr.org/2016/01/how-subscriptions-are-creatingwinners-and-losers-in-retail

Seamans, R., \& Zhu, F. (2014). Responses to entry in multi-sided markets: The impact of Craigslist on local newspapers. Management Science, 60(2), 476-493. DOI: https://doi.org/10.1287/mnsc.2013.1785

Sibata, K. T. (2017). Segmentação de Clientes de uma Empresa de Assinatura através da análise de clusters. Trabalho de Conclusão de Curso (Bacharel em Engenharia de Produção). Universidade de São Paulo.

Taylor, S., \& Bogdan, R. (1992). Introducción a la observación participante. Barcelona. Editorial Paidos. 